\title{
Glatiramer acetate treatment effects on gene expression in monocytes of multiple sclerosis patients
}

\author{
Madhan Thamilarasan ${ }^{1,2 \dagger}$, Michael Hecker ${ }^{1,2^{*}{ }^{\dagger}}$, Robert Hermann Goertsches ${ }^{1,2}$, Brigitte Katrin Paap ${ }^{2}$, Ina Schröder ${ }^{2}$, \\ Dirk Koczan ${ }^{1}$, Hans-Jürgen Thiesen ${ }^{1}$ and Uwe Klaus Zettl ${ }^{2}$
}

\begin{abstract}
Background: Glatiramer acetate (GA) is a mixture of synthetic peptides used in the treatment of patients with relapsing-remitting multiple sclerosis (RRMS). The aim of this study was to investigate the effects of GA therapy on the gene expression of monocytes.

Methods: Monocytes were isolated from the peripheral blood of eight RRMS patients. The blood was obtained longitudinally before the start of GA therapy as well as after one day, one week, one month and two months. Gene expression was measured at the mRNA level by microarrays.

Results: More than 400 genes were identified as up-regulated or down-regulated in the course of therapy, and we analyzed their biological functions and regulatory interactions. Many of those genes are known to regulate lymphocyte activation and proliferation, but only a subset of genes was repeatedly differentially expressed at different time points during treatment.

Conclusions: Overall, the observed gene regulatory effects of GA on monocytes were modest and not stable over time. However, our study revealed several genes that are worthy of investigation in future studies on the molecular mechanisms of GA therapy.
\end{abstract}

Keywords: Glatiramer acetate, Relapsing-remitting multiple sclerosis, Monocytes, Gene expression profiling, Microarray analysis

\section{Background}

Multiple sclerosis (MS) is a chronic immune-mediated disease of the central nervous system (CNS). The autoreactive behavior of the immune system in MS patients is associated with inflammatory lesions in the CNS and axonal demyelination. There is currently no cure for MS, but there are several therapies available as diseasemodifying agents. The relapsing-remitting type of MS (RRMS) is mainly treated with immunomodulating drugs like interferon-beta (IFN- $\beta$ ) and glatiramer acetate (GA) [1,2].

\footnotetext{
* Correspondence: michael.hecker@rocketmail.com

${ }^{\dagger}$ Equal contributors

${ }^{1}$ Institute of Immunology, University of Rostock, Schillingallee 68, Rostock 18057, Germany

${ }^{2}$ Department of Neurology, Division of Neuroimmunology, University of Rostock, Gehlsheimer Straße 20, Rostock 18147, Germany
}

GA (copolymer 1) is a first-line treatment option for RRMS. Different clinical trials have shown that GA treatment decreases the incidence of relapses and significantly reduces the number of gadolinium-enhancing lesions in magnetic resonance imaging (MRI) [3-6]. GA is not a defined chemical substance, but a standardized mixture of synthetic peptides. These peptides are made up of four different amino acids, glutamic acid, lysine, alanine and tyrosine (G-L-A-T), in a molar ratio of 1.5:3.6:4.6:1.0, assembled in a random order into polypeptide chains with a length of 40 to 100 residues [7]. This mixture of peptides was initially intended to mimic myelin basic protein (MBP) and to induce experimental autoimmune encephalomyelitis (EAE), the animal model of MS [8]. However, surprisingly, GA inhibited EAE in rodents and monkeys [9]. Today, GA has been well-

\section{Biomed Central}

(c) 2013 Thamilarasan et al.; licensee BioMed Central Ltd. This is an open access article distributed under the terms of the Creative Commons Attribution License (http://creativecommons.org/licenses/by/2.0), which permits unrestricted use, distribution, and reproduction in any medium, provided the original work is properly cited. 
established for MS therapy for more than a decade due to its beneficial clinical effects and its favorable safety profile.

According to pharmacokinetic studies, GA is quickly absorbed after subcutaneous administration, and it undergoes rapid degradation to amino acids and shorter peptides. Only $10 \%$ of the peptides remain at the site of injection after one hour [10]. A fraction of GA presumably enters the lymphatic circulation and reaches the regional lymph nodes where it modulates immune responses. Of note, patients treated with GA have the tendency to develop antibodies against it. However, the biological meaning of anti-GA antibodies remains controversial and it is unclear whether they may have a neutralizing or a beneficial effect in MS patients [11-14].

Different molecular mechanisms of action of GA have been proposed [7,15-18]. One postulated mechanism is that GA peptides act as altered peptide ligands (APL). An APL is a peptide, usually closely related to an agonist peptide in amino acid sequence, that induces a different function or partial response of T-cells specific for the agonist peptide as a result of the modified interaction with the T-cell receptor (TCR). During GA therapy, the partial activation of T-cells specific for MBP and other myelin antigens can induce peripheral tolerance, and this may contribute to the clinical effects of GA by preventing the attack of the myelin sheath around the nerves. Indeed, cross-reactivity of GA-specific T-cells with myelin antigens has been demonstrated, and there is evidence that prolonged exposure to GA results in anergy or depletion of GA-reactive cells that are possibly relevant in the pathogenesis of MS [19-23]. On the other hand, several studies have shown that GA induces a T helper cell type 1 (Th1) to Th2 shift in T-cells. Th1 and Th2 each produce a different combination of pro- and anti-inflammatory cytokines, respectively. The increase of GA-reactive Th2 cells during treatment is regarded as a central mechanism of action of GA [24-27]. These anti-inflammatory Th2-like cells were found to mediate regulatory functions as they can migrate into the brain and act suppressively at the sites of inflammation (local bystander suppression). This leads to a reduced activation and proliferation of auto-reactive immune cells, even if they recognize unrelated antigens and do not cross-react with GA [11].

Antigen-presenting cells (APC) are also believed to play a role in the immunomodulatory effects of GA therapy. The professional APC are dendritic cells and macrophages, which differentiate from circulating monocytes [28]. The interplay between APC and T-cells is fundamental in adaptive immune responses as well as in the pathophysiology of MS [29]. One suggested mechanism of GA is that it binds to major histocompatibility complex (MHC) molecules and thus competes with myelin antigens for their presentation on APC to T-cells. Specifically,
GA can act as an antagonist of MBP/MHC at MBPspecific TCR and it is able to displace MBP from the binding site on MHC class II molecules [30,31]. On the other hand, GA was shown to change the properties of APC in such a way that they stimulate Th2-like responses. These APC are called type II APC. The effect of the drug on APC seems to depend on the cell type [11,32]. Weber et al. showed that GA inhibits monocyte reactivity and induces type II monocytes, which promote both Th2 differentiation and expansion of $\mathrm{T}$ regulatory cells (Treg). They observed that after GA administration in EAE, the pattern of cytokine production by monocytes switched towards an anti-inflammatory profile, characterized by down-regulation of pro-inflammatory cytokines (for example, IL12) and up-regulation of anti-inflammatory cytokines (for example, IL10) [33,34]. An increased IL10 production of monocytes has already been observed 72 hours post GA therapy initiation in a recent study by Ayers et al. [35]. Other studies confirmed that there is an increase in anti-inflammatory type II monocytes during GA therapy, and that the suppressor functions of these monocytes contribute to Th2 deviation of naive T-cells of MS patients [36-38]. Additionally, GA was shown to affect monocytes by increasing the expression of IL1RA while diminishing the production of IL1- $\beta[39,40]$. Recently, Caragnano et al. also observed a trend for IL1- $\beta$ downregulation in stimulated monocytes from GA-treated MS patients, and this was paralleled by lower levels of P2RX7, a receptor regulating cytokine production and apoptosis [41]. However, whether GA acts directly on monocytes in vivo, or whether the effects on monocytes are mediated by cytokines produced by GA-specific Th2 cells, is unclear as there is a dynamic feedback loop between human T-cell and APC responses [11,36].

However, GA not only modulates CD4+ T helper cell responses and binds to MHC class II molecules on APC. It has also been shown that GA incites an HLA class Irestricted, cytotoxic suppressor CD8+ T-cell response [42]. This may be mediated by heat shock proteins (HSPs) that bind extracellular antigens and mediate their cellular uptake. HSP-antigen complexes are then directed toward either the conventional class II pathway or the MHC class I pathway through cross-presentation $[43,44]$. In the similar way, GA peptides may bind to HSPs, and thus may be presented on MHC class I molecules resulting in an altered activation of T-cell subsets. This potentially leads to cytotoxic T-cells, which can kill CD4+ T-cells in a GA-specific manner [42]. In addition to its immunomodulatory effects, direct neuroprotective and even remyelinating properties have been ascribed to GA as well [45-49]. For instance, GA may foster repair after neurologic damage by stimulating the expression of neurotrophic factors like BDNF by various immune and CNS resident cells [50]. 
Over the last years, several research groups performed longitudinal gene expression profiling studies with microarrays to better understand the mechanisms of action of MS therapies. However, while the broad and rapid gene regulatory effects of IFN- $\beta$ treatment in blood cells have been investigated extensively [51], there is only one such study for GA treatment: Achiron et al. measured the gene expression in peripheral blood mononuclear cells (PBMC) from 14 RRMS patients before and three months after initiation of GA therapy [52]. In their analysis, they identified 480 genes to be differentially expressed at the transcript level. They concluded that changes in the expression of immunomodulatory genes during GA therapy are important to reduce the activity of the disease [52].

The present study focuses on the effects of daily subcutaneous GA injections on the mRNA expression profile of monocytes in the peripheral blood. We were interested in monocytes because GA has been described as modulating these cells to promote Th2-like responses $[11,31]$, but the in vivo effects have so far not been examined in a genome-wide and longitudinal manner. We obtained monocytes from RRMS patients immediately before as well as at four different time points after the start of GA therapy. The gene expression analysis was performed using microarrays. Genes that were found to be differentially expressed in response to GA therapy were then analyzed for biological functions and molecular interactions to derive new hypotheses on the molecular mechanisms of action of GA. This is the first study that investigates the transcriptome dynamics over the course of the therapy in a cell type-specific manner.

\section{Methods}

\section{Blood sample collection}

Eight Caucasian patients with diagnosed RRMS according to the revised McDonald criteria [53] were recruited for this study. The patients started a treatment with GA
(Copaxone, Teva Pharmaceutical Industries Ltd., Petah Tikva, Israel) in $20 \mathrm{mg}$ doses given daily as a subcutaneous injection. Five of the patients were not treated with any immunomodulatory or immunosuppressive drug prior to the onset of this study. Two patients (MS3 and MS5) received subcutaneous IFN- $\beta$, and one patient (MS8) received mitoxantrone (the last injection was four months ago) previously (Table 1). All patients were given routine care following the consensus treatment guidelines and recommendations of the German Society of Neurology (DGN). Blood samples were obtained from each patient at five different time points: before the first injection of GA (baseline) and after one day (that is, before the second injection) as well as after one week, one month and two months. Collection of blood was done by venipuncture with ethylenediaminetetraacetic acid (EDTA) as anticoagulant. An approximate volume of $15 \mathrm{ml}$ whole blood was collected for each patient and each time point. In the clinical follow-up, the patients were assessed neurologically, monitored for relapses, and rated using the expanded disability status scale (EDSS) and cranial MRI. The study was approved by the ethics committee of the University of Rostock and carried out according to the Declaration of Helsinki. All patients gave written informed consent to be included in the study.

\section{Monocyte isolation, RNA preparation and gene expression profiling}

Monocytes were isolated from the blood samples using erythrocyte lysis buffer (Qiagen, Hilden, Germany), followed by magnetic-activated cell sorting (MACS). The CD14+ cells were magnetically labeled using CD14 MicroBeads and collected as positively selected cell fraction using the autoMACS Separator (Miltenyi Biotec, Teterow, Germany). Total RNA was then isolated from the monocytes using RNeasy columns (Qiagen, Hilden, Germany). RNA concentrations were measured using a NanoDrop 1000 spectrophotometer (Thermo Fisher Scien-

Table 1 Demographic data and clinical data of the eight patients

\begin{tabular}{|c|c|c|c|c|c|c|c|c|}
\hline Patient & Gender & $\begin{array}{c}\text { Age at } \\
\text { study onset }\end{array}$ & $\begin{array}{l}\text { Disease } \\
\text { duration }\end{array}$ & $\begin{array}{l}\text { Previous } \\
\text { treatment }\end{array}$ & $\begin{array}{l}\text { EDSS at } \\
\text { baseline }\end{array}$ & $\begin{array}{l}\text { EDSS after } \\
12 \text { months }\end{array}$ & $\begin{array}{l}\text { Relapses during first } \\
12 \text { months }\end{array}$ & $\begin{array}{c}\text { CMRI at } \\
\text { follow-up }\end{array}$ \\
\hline MS1 & Female & 41 & 0 & None & 1.5 & 1.5 & 0 & - \\
\hline MS2 & Female & 50 & 10 & None & 1.5 & 1.5 & 1 & New lesion \\
\hline MS3 & Female & 37 & 33 & IFN-beta sc. & 1.0 & 1.5 & 0 & stable \\
\hline MS4 & Female & 38 & 9 & None & 1.0 & 1.5 & 0 & New lesion \\
\hline MS5 & Female & 38 & 89 & IFN-beta sc. & 3.5 & 2.0 & 1 & stable \\
\hline MS6 & Female & 47 & 1 & None & 1.5 & 1.5 & 0 & New lesion \\
\hline MS7 & Male & 35 & 16 & None & 1.0 & 1.0 & 0 & New lesion \\
\hline MS8 & Male & 25 & 55 & Mitoxantrone & 2.0 & 2.5 & 1 & New lesion \\
\hline
\end{tabular}

The table shows gender, age, the previous treatment, and the EDSS scores at therapy initiation (baseline) and after 12 months. The duration from the diagnosis of definite MS to the start of GA therapy in months (disease duration) is also given. The gender ratio was 6:2 (female: male) and the average age at study onset was $38.9 \pm 7.6$ years (mean \pm SD). Three patients had a relapse during the first 12 months after GA treatment initiation. Five patients showed a new brain lesion in the follow-up compared to pre-treatment. cMRI: cranial magnetic resonance imaging, EDSS: expanded disability status scale, sc.: subcutaneous, SD: standard deviation. 
tific, Wilmington, DE, USA) and their integrity was assessed with an Agilent 2100 Bioanalyzer using RNA 6000 Pico LabChips. The microarray experiments were performed with an Affymetrix platform. HG-U133 Plus 2.0 GeneChips were used for this analysis. From each sample preparation, total RNA amounts ranging from $100 \mathrm{ng}$ to $200 \mathrm{ng}$ were used as starting material. RNA was converted to cDNA and later into biotinylated cRNA using the MessageAmp II-Biotin Enhanced Kit (Ambion, Foster City, CA, USA). The cRNA molecules were fragmented and $15 \mu \mathrm{g}$ of cRNA were hybridized onto the GeneChips for 16 hours at $45^{\circ} \mathrm{C}$. The GeneChips were later washed and stained in the Affymetrix Fluidics Station 450 and scanned with a GeneChip Scanner $30007 \mathrm{G}$ system. All these procedures were performed according to the manufacturer protocols (Affymetrix, Santa Clara, CA, USA).

\section{Microarray data pre-processing}

Initial data pre-processing and quality control was done using the Affymetrix GeneChip operating software (GCOS 1.4) and MAS5.0 statistical algorithms (Microarray Analysis Suite 5.0) (Affymetrix, Santa Clara, CA, USA). Since the annotation of genes has changed since the development of the microarrays, there are oligonucleotide probes on the chips, which match to no transcript, and probes, which match to multiple transcripts. Therefore, to exclude such probes from the analysis, we used a custom chip definition file (CDF), which was based on the GeneAnnot database version 1.9 (http://www.xlab. unimo.it/GA_CDF/, CDF version 2.1.0) [54]. Each probe set in the custom CDF matches a single gene. Data normalization was done by a loess fit to the data with span $=0.05$ using the R package 'affy'.

\section{Filtering of differentially expressed genes}

To filter differentially expressed genes from the data, we applied two criteria. First, we computed paired $t$-tests comparing for each gene the expression at baseline with the expression at one day, one week, one month and two months. In the second analysis, we evaluated the data with the MAID filtering method, which calculates MA plot-based signal intensity-dependent fold-changes (MAID-scores) for each time point comparison [55]. To filter genes that are significantly up-regulated or down-regulated relative to baseline, we combined the MAID-score outcomes with the paired $t$-test outcomes. Genes with $\mid$ MAID-score $\mid>2$ and $t$-test $P$-value $<0.05$ were filtered as differentially expressed.

\section{Functional analysis of differentially expressed genes}

To investigate the functions of the filtered genes, we performed a Gene Ontology (GO) term enrichment analysis, which was based on the association of functional annotations for each gene in the GO database. GOstats [56], a Bioconductor software package, was the application used to test GO terms for overrepresentation. GOstats computes a probability based on a hypergeometric distribution, which assesses whether the number of filtered genes associated with the term is larger than expected by chance. As a reference, that is, the gene universe, we used all genes that were measured with the HG-U133 Plus 2.0 microarrays $(\mathrm{n}=18,862)$.

\section{Text mining-based gene interaction network analysis}

A gene interaction network was constructed for the filtered genes using the Pathway Studio software version 7.1 from Ariadne Genomics (Rockville, MD, USA) [57]. This software allowed the extraction of gene interactions that were automatically obtained from the literature by text mining. The information about interactions between the genes was exported from the Pathway Studio software as a table, which contains nodes (genes) and edges (interactions) as the building blocks of the gene network. Cytoscape, which is an open source software [58], was used to visualize the network. The edges were represented in various shapes to display the type of the interaction (positive, inhibitory and binding).

\section{Results}

\section{Patient and sample information}

Eight patients were included in this study (six females and two males). The patients were $38.9 \pm 7.6$ years of age (mean \pm standard deviation) and had a mean EDSS of 1.6 (1.0 to 3.5) after a mean disease duration of 26.6 (0 to 89) months (Table 1). All patients started GA treatment at standard dose. During the follow-up period of 12 months, three patients had one relapse each and the other five patients had no relapse. There was only a moderate increase in disability when comparing the EDSS at study onset (baseline) with the EDSS after a follow-up of one year (Table 1). One patient discontinued the therapy in this period of time: MS5 had a severe relapse soon after study onset and, therefore, switched to natalizumab therapy (Tysabri, Biogen Idec, Weston, MA, USA) after three months. Cranial MRI scans were done for seven of the eight patients before the start of GA therapy as well as after a mean follow-up of $17.6 \pm 9.5$ months. Despite the therapy, five patients each had one new lesion.

From each blood sample, monocytes were isolated by MACS separation. For quality control, we analyzed the mRNA levels of genes, which are known to be specifically expressed by different blood cell types [59]. This revealed high CD14+ monocyte purities (Additional file 1: Figure S1). The RNA that was isolated from the monocytes was in general of high quality with an average RNA integrity number (RIN) of 9.6. The quality of RNA was poor for three samples (MS3/1 month, MS6/1 week, 
MS7/1 week) and so they were excluded from further analyses.

\section{Differentially expressed genes}

The pre-processing of the Affymetrix microarray data resulted in transcript levels for 18,862 different genes and 37 different samples. The data are available in the Gene Expression Omnibus (GEO) database with the accession number 'GSE42763'. The filtering of genes differentially expressed in response to GA therapy resulted in a gene list for each time point comparison. In total, 171 genes met the filtering criteria for one day, 116 genes for one week, 124 genes for one month and 101 genes for two months versus baseline (Figure 1, Additional file 2: Table S1). These four gene lists, when aggregated, resulted in 463 different genes (293 up-regulated and 170 down-regulated genes). We observed no accumulation of gene regulatory effects in the course of the therapy since similar numbers of genes were filtered at early (within the first week) and later time points (after one and two months). Moreover, unexpectedly, we found no stable signature of GA-responsive genes since only 45 of the 463 genes were repeatedly identified as differentially expressed, and there was no gene modulated in expression at all time points during therapy.

To further narrow down the list of genes, we selected only those genes that were expressed at significantly higher or lower levels compared to baseline at two or more consecutive time points. This resulted in a subset of 23 out of the 463 genes. Of these 23 genes, 5 genes were down-regulated (CD34, RPA4, HMGB1L4, BAZ2B and RARS) and 18 genes were up-regulated (for example, ATOX1, BLOC1S1, LIMD2, POLR2I and RPA3). The average mRNA expression dynamics of these genes during GA therapy are shown in Figure 2.

\section{Functional annotation of the genes}

A Gene Ontology (GO) term enrichment analysis was performed to determine the functions, which are characteristic for the genes filtered as differentially expressed in response to GA therapy $(\mathrm{n}=463)$. The GO terms are classified into three major categories: biological process (BP), cellular component (CC) and molecular function (MF). In our analysis, we shortlisted the top 20 overrepresented GO terms according to the $P$-value (Table 2). Several genes appeared under multiple GO terms. The GO terms, which had the most gene members in the list of filtered genes, were 'extracellular region' (GO:0005576, $P$-value $=2.27 \mathrm{E}-07$ ) and 'immune system process' (GO:0002376, $P$-value $=1.19 \mathrm{E}-04)$. The GO term 'extracellular region' contains genes whose protein products are secreted from cells, for example, cell communication molecules, and it was associated to 76 of the 463 genes. Out of them, 45 genes were up-regulated, for example, POMC, MMP17, LTB, XCL1 and APOL3, and 31 genes were down-regulated, for example, CD163, ADAMTS5, TNFSF14, CTSZ and PAM. The GO term 'immune system process' contained 48 of the genes. Out of them, 35 genes were up-regulated, for example, CD38, CXCL9, CXCL10, IL18 and ICAM2, and 13 genes were down regulated, for example, PTPRC, NCK2, C4BPA,
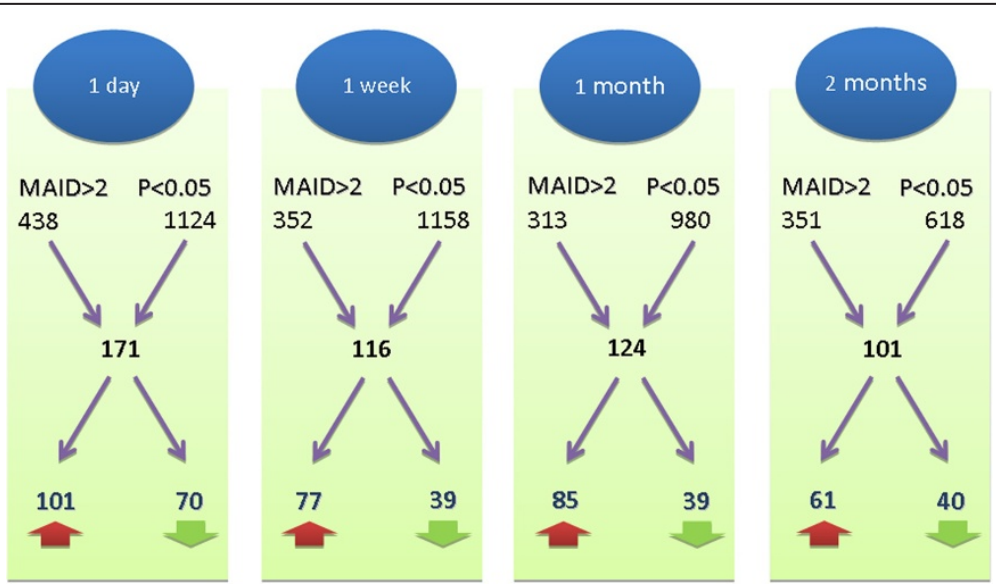

Figure 1 Longitudinal study design and gene filtering results. Blood was sampled from eight patients at five different time points: before the start of GA treatment as well as after one day, one week, one month and two months. The expression of 18,862 genes was measured in monocytes with Affymetrix microarrays, and transcript levels during therapy were compared to the pre-treatment levels. $t$-test $P$-values and MAID-scores were used to determine differentially expressed genes. For instance, when comparing the baseline levels with the expression levels after one day, 438 genes survived the MAID analysis criterion (|MAID-score $\mid>2$ ) and 1,124 genes survived the paired $t$-test criterion $(P$-value $<0.05)$. In combination, this resulted in 171 filtered genes: 101 were up-regulated (red arrow) and 70 were down-regulated (green arrow). For each time point comparison, a similar number of genes were filtered. However, the overlap of these four gene lists was relatively small $(n=45)$. When taken together, 463 different genes were identified to be differentially expressed within the first two months of GA therapy. 


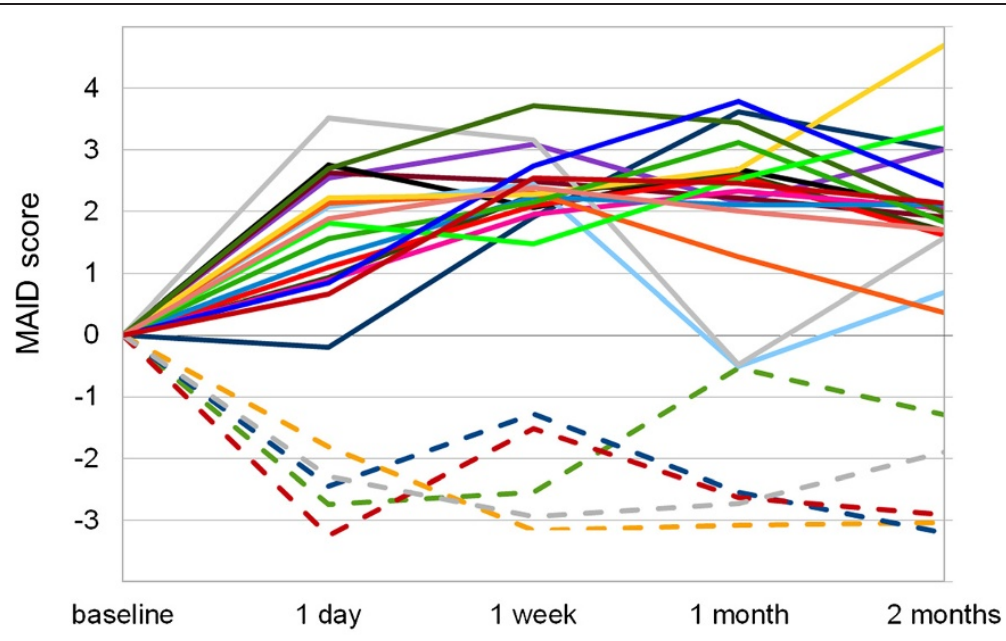

$$
\begin{array}{|llll|}
\hline \text { APCDD1 } & - \text { ATOX1 } & - \text { ATP5I } & - \text {-BAZ2B } \\
\text { BLOC1S1 } & - \text { C11orf84 } & - \text { C12orf57 } & - \text { C17orf61 } \\
\text { C7orf47 } & -{ }_{\text {CD34 }} & - \text { CD68 } & - \text { CD72 } \\
-- \text { HMGB1L4 } & - \text { KLRF1 } & - \text { LIMD2 } & - \text { MMP17 } \\
- \text { POLR21 } & - \text { PTK6 } & - \text { PTPRCAP } & - \text {-RARS } \\
\text { RPA3 } & - \text { - RPA4 } & - \text { RPPH1 } & \\
\hline
\end{array}
$$

Figure 2 Dynamics of 23 genes consistently modulated in expression during glatiramer acetate (GA) therapy. A subset of the 463 filtered genes appeared repeatedly as differentially expressed in consecutive time point comparisons. The graph shows the mean mRNA expression dynamics of 23 genes that were repeatedly found expressed at significantly higher or lower levels across at least two different time points compared to the pre-treatment levels. There were 18 up-regulated genes and 5 down-regulated genes (CD34, RPA4, HMGB1L4, RARS and BAZ2B). The MAID-score, a fold-change variant that is calculated from the data of all patients, is represented on the $y$-axis and the time points are represented on the $x$-axis.

GLMN and ITGAV. There were 22 genes that belonged to both of these GO terms.

There were several overrepresented GO terms, which are relevant to monocytes, for instance 'mononuclear cell proliferation' (GO:0032943, $P$-value $=5.76 \mathrm{E}-04)$, 'regulation of mononuclear cell proliferation' (GO:0032944, $P$-value $=5.44 \mathrm{E}$-04) and 'leukocyte proliferation' (GO:00 70661, $P$-value $=6.92 \mathrm{E}-04)$. These GO terms form a hierarchy where a $\mathrm{GO}$ term is part of a broader $\mathrm{GO}$ term, hence these GO terms share most of the genes. Genes found modulated in expression during GA treatment and belonging to all of these three terms are, for example, CD38, GLMN, IGHM, IL18, NCK2 and PTPRC. Other notable overrepresented GO terms were 'regulation of lymphocyte activation' and 'cytokine activity'.

\section{Gene interaction network}

The unified list of 463 genes was used as input for the Pathway Studio software to gather pair-wise interactions between them. The output resulted in 41 genes (= nodes) with 59 interactions (= edges). The interactions were visualized as a network (Figure 3). The edges vary according to their interaction type. There were 43 positive regulatory interactions, 14 inhibitory interactions and 2 binding interactions. The network revealed different interaction clusters. Seven of the genes, CXCL10, CXCL9, VCAM1, POMC, OXT, PTPRC and CD38, possess the majority of the interactions with the other genes in the network: except PTPRC, they all appeared as up-regulated during GA therapy.

POMC, a polypeptide hormone precursor, had 12 interactions and is therefore the most connected gene in the network. For instance, VCAM1, OXT, IL18 and ADCY6 (which were up-regulated) and IGFBP1 (which was down-regulated) are influenced by POMC according to the literature-based interaction network. The second cluster is based on VCAM1, a vascular cell adhesion protein, which had 11 interactions. It is regulated by IL18, POMC, ITGAV, CYP2C19, CXCL10, CXCL9, TRAF2, NOTCH4 and TIE1. Of these, ITGAV was down-regulated and all the other genes were up-regulated in response to GA treatment. The third cluster is formed by OXT, which regulates CD38, SLA5A5, GHRH and POMC. The fourth cluster is based on two up-regulated chemokines, CXCL9 and CXCL10, which together had 14 interactions. They have a feedback loop between them, and CXCL10 is further regulated by IL18, IL27 and XCL1, which were all up-regulated after the first week of GA therapy. The network also shows that PTPRC $(=$ CD45) is linked with PTPRCAP, CD34, CD38, IL21 and VCAM1. 
Table 2 Analysis of gene functions

\begin{tabular}{|c|c|c|c|c|c|}
\hline Term & GO accession & ExpCount & Count & Odds ratio & $P$-value \\
\hline Extracellular region & GO:0005576 (CC) & 42 & 76 & 2.06 & 2.27E-07 \\
\hline Cytokine activity & GO:0005125 (MF) & 4 & 15 & 4.18 & $1.12 \mathrm{E}-05$ \\
\hline Receptor binding & GO:0005102 (MF) & 21 & 41 & 2.19 & $1.85 \mathrm{E}-05$ \\
\hline Extracellular region part & GO:0044421 (CC) & 22 & 42 & 2.06 & 5.17E-05 \\
\hline Immune response & GO:0006955 (BP) & 17 & 35 & 2.19 & $6.74 \mathrm{E}-05$ \\
\hline Extracellular space & GO:0005615 (CC) & 17 & 34 & 2.17 & $9.00 \mathrm{E}-05$ \\
\hline Immune system process & GO:0002376 (BP) & 28 & 48 & 1.90 & 1.19E-04 \\
\hline Regulation of lymphocyte activation & GO:0051249 (BP) & 5 & 14 & 3.30 & 2.19E-04 \\
\hline Defense response & GO:0006952 (BP) & 18 & 33 & 2.01 & 4.38E-04 \\
\hline Regulation of lymphocyte proliferation & GO:0050670 (BP) & 2 & 9 & 4.27 & 5.07E-04 \\
\hline Lymphocyte proliferation & GO:0046651 (BP) & 3 & 10 & 3.89 & $5.08 \mathrm{E}-04$ \\
\hline T-cell proliferation & GO:0042098 (BP) & 2 & 8 & 4.75 & 5.37E-04 \\
\hline Regulation of mononuclear cell proliferation & GO:0032944 (BP) & 2 & 9 & 4.23 & 5.44E-04 \\
\hline Cytokine receptor binding & GO:0005126 (MF) & 4 & 12 & 3.33 & $5.48 \mathrm{E}-04$ \\
\hline Mononuclear cell proliferation & GO:0032943 (BP) & 3 & 10 & 3.82 & $5.76 \mathrm{E}-04$ \\
\hline Regulation of leukocyte activation & GO:0002694 (BP) & 5 & 14 & 2.97 & $5.90 \mathrm{E}-04$ \\
\hline Regulation of leukocyte proliferation & GO:0070663 (BP) & 2 & 9 & 4.14 & $6.26 \mathrm{E}-04$ \\
\hline Leukocyte proliferation & GO:0070661 (BP) & 3 & 10 & 3.72 & $6.92 \mathrm{E}-04$ \\
\hline Regulation of cell activation & GO:0050865 (BP) & 5 & 14 & 2.82 & $9.40 \mathrm{E}-04$ \\
\hline Regulation of T-cell proliferation & GO:0042129 (BP) & 2 & 7 & 4.93 & $9.56 \mathrm{E}-04$ \\
\hline
\end{tabular}

The top 20 Gene Ontology (GO) terms that were significantly overrepresented for the list of filtered genes ( $n=463$ genes) are shown in the table sorted by $P$-value. For example, in the second row, 'cytokine activity' is listed as an overrepresented GO term. 'GO:0005125' is the corresponding GO database accession. The third and fourth column give the expected number ('ExpCount', 4 genes) and the actual number ('Count', 15 genes) of genes in the filtering result that are associated with 'cytokine activity'. This led to an odds ratio of 4.18 and a $P$-value of $1.12 \mathrm{E}-05$. The GO terms are classified into three major groups: biological process (BP), cellular component (CC) and molecular function (MF).

CD38 had six interactions: CD38 inhibits CD34, is regulated by IL21, and has feedback loops with OXT and PTPRC (Figure 3).

\section{Discussion}

This study focused on the in vivo effects of GA therapy on the gene expression of monocytes, which are the precursors of macrophages and dendritic cells. The monocytes were obtained from the blood of RRMS patients and we compared the transcript levels before and after the start of GA therapy. For each patient, the gene expression was measured at five time points up to two months into therapy. In these data, we identified 463 genes as up-regulated or down-regulated during therapy compared to pre-treatment levels. More than a hundred genes were filtered at early (after one day) and later (after two months) time points post treatment initiation. However, relatively few genes were repeatedly found to be differentially expressed in the course of time. This indicated that the gene regulatory effects of GA on monocytes are rather modest and no stable gene expression signature could be seen. Nevertheless, the mRNA changes of some genes might tell us something about GA's molecular mechanisms of action.
Compared to our study, in the gene expression study by Achiron et al., only two time points were compared: before and after three months of GA treatment [52]. Therefore, the variability in the mRNA dynamics early during therapy possibly has been underestimated so far. Moreover, Achiron et al. studied the gene expression changes in PBMC of RRMS patients, whereas we studied monocytes. Using Affymetrix microarrays, they found 480 genes to be differentially expressed in response to GA administration, with the main effects being related to antigen-activated apoptosis, inflammation, adhesion, and MHC class I antigen presentation [52]. As in our study, there were more up-regulated than down-regulated genes. However, when comparing their gene list $(n=480)$ with ours $(n=463)$, only five genes (BAT1, ELOVL5, ETV7, MT1E and PCBD1) were in common. One explanation for that might be that GA possibly acts primarily on other subsets of circulating cells, for example, by altering the functional properties of (autoreactive) T-cells. Therefore, different gene regulatory effects might be seen in PBMC than in monocytes from GA-treated patients.

On the other hand, a recent cross-sectional study by Ottoboni et al. found no significant differences in the PBMC RNA profiles of untreated and GA-treated patients 


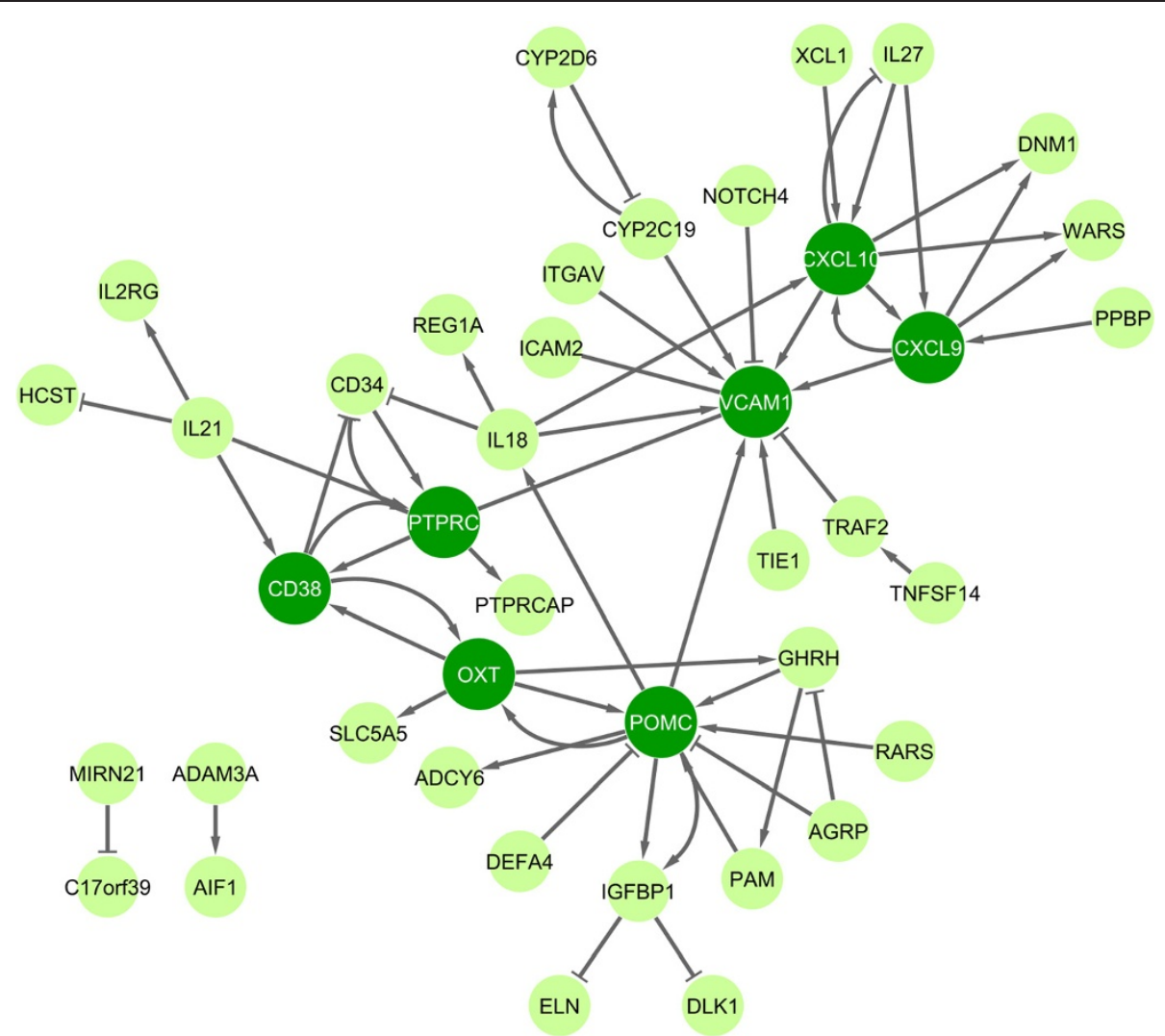

Figure 3 Interaction network of genes differentially expressed in response to glatiramer acetate (GA). A gene interaction network was retrieved for the 463 filtered genes using the Pathway Studio software. The software delivered a network of 41 genes (= nodes) with 59 interactions (= edges) between them. The other genes with no interaction are not shown in the figure. Edges are represented in different ways in the network. An arrow $(\rightarrow)$ means positive effect $(n=43)$, a 'T' $(-t)$ means negative effect (inhibition, $n=14)$ and a line $(-)$ means binding $(n=2)$. The seven nodes, which are highlighted in dark green, are involved in the regulation of many other genes and thus may play an important role in the molecular mechanisms of action of GA. The network is available as a Cytoscape session file from the authors upon request.

[60]. In their study, they took into account the multiple testing by computing false discovery rates (FDR) [61]. In our data set, if we set the threshold for statistical significance at $\mathrm{FDR}<0.05$, also no gene remained as differentially expressed during GA therapy. Instead, we chose less conservative filtering criteria to detect even moderate shifts in the gene expression of monocytes, but, in consequence, also some weakly modulated and less expressed genes survived the filtering for GA-responsive genes (Additional file 2: Table S1). A subset of 23 genes was repeatedly identified to be up-regulated or downregulated at different time points during therapy (Figure 2). These 23 genes might represent good candidates of molecular markers of GA activity. However, for confirmation, a larger independent study with more sensitive measurement techniques such as real-time PCR is needed. As another limitation of our study, we did not measure the transcript levels in the long-term after the first two months of treatment. Possibly, the full modulation of immunological processes by GA may require more time. It was also beyond the scope of the present study to examine whether the individual gene expression profiles are associated with the clinical data (for example, relapse rate, EDSS and MRI).

We performed a GO term enrichment analysis for the list of 463 filtered genes to classify them according to their functions and the biological processes they are involved in (Table 2). Overrepresented GO terms included 'lymphocyte proliferation' and 'regulation of T-cell proliferation'. These findings are consistent with earlier studies that showed that GA suppresses lymphocyte proliferation through modulation of monocytes and monocyte-derived dendritic cells, thereby reducing the number of autoreactive T-cells $[38,62,63]$. Members of these GO terms are, for example, cytokines such as IL18 and TNFSF14. Additionally, we searched for interactions between the genes and retrieved 59 interactions. Of note, these interactions were obtained by literature mining. Therefore, the gene network (Figure 3) shows direct as well as indirect regulatory effects on the transcript and protein level. Seven genes had several interactions (CXCL9, CXCL10, VCAM1, POMC, OXT, PTPRC and CD38). 
The network contained different cytokines, for example, CXCL9 and CXCL10 whose expression was increased one day after the start of GA therapy. These two chemokines bind to the CXCR3 receptor and are involved in the recruitment of immune cells to sites of inflammation, principally acting on activated CD4+ Th1 cells, CD8+ T-cells and natural killer (NK) cells $[64,65]$. A previous study already showed that the transcription of CXCL10 is induced in PBMC after GA administration [66]. Both CXCL9 and CXCL10 were also described to be modulated in expression in blood during treatment with IFN- $\beta$ [67]. Therefore, both drugs seem to affect the chemokine gradient between brain lesions and the peripheral immune compartment. The cytokine group further included interleukins (IL18, IL21, IL25 and IL27), which were all found to be up-regulated in our data set. Of those, IL18 and IL27 play important roles in the differentiation and expansion of naive CD4+ T-cells [68,69]. Moreover, TNFSF14, a member of the TNF cytokine family, was down-regulated one day after the first GA injection. TNFSF14 is known to function as a costimulatory factor regulating the activation of T-cells [70]. A single nucleotide polymorphism within an intron of the TNFSF14 gene is associated with MS susceptibility [71,72]. Another related member of the TNF family, LTB (lymphotoxin- $\beta$ ), was also filtered as differentially expressed. Both LTB and TNFSF14 bind to the LTBR receptor, and they provide communication links in innate and adaptive immune responses $[73,74]$. However, the transcriptional modulation of these cytokines was not stable over time and it thus remains unclear how these immunoregulatory effects may exactly contribute to the mechanisms of action of GA.

The interaction network of filtered genes also contains several cell adhesion receptors, for example, ITGAV, ICAM2 and VCAM1. ITGAV encodes the integrin $\alpha \mathrm{V}$, an integral membrane protein that can interact with a variety of extracellular matrix ligands. Integrins orchestrate monocyte differentiation into macrophages, and they play a role in macrophage adhesion, migration and tissue infiltration [75]. Moreover, ITGAV is known to mediate proinflammatory cytokine synthesis in human monocytes [76]. It was expressed at lower levels after the first injection of GA, which may reflect the previously described shift in the gene expression of monocytes towards an anti-inflammatory profile [34,39].

Other genes in the network are implicated in quite different biological processes, for example, RARS, WARS, PTPRC, PTPRCAP and MIRN21. RARS and WARS encode the arginyl- and tryptophanyl-tRNA synthetase, respectively. They catalyze the amino acid attachment to cognate tRNAs during protein synthesis. However, besides their role in protein translation, biologically active fragments of WARS were also discovered to be involved in angiogenesis signaling pathways [77]. PTPRC is a transmembrane glycoprotein associated with PTPRCAP. Both genes were found modulated in expression after one month of treatment compared to baseline. PTPRC functions as a regulator of cytokine receptor signaling and influences cellular processes such as cell proliferation [78]. Upon activation of monocytes, proteolytic processing of PTPRC results in a protein fragment, which is released and acts as an inhibitor of T-cell proliferation [79]. The microRNA gene MIRN21 was expressed at higher levels after two months compared to pre-treatment levels. MicroRNAs are involved in the post-transcriptional regulation of gene expression. The transcript MIRN21 harbors the mature microRNA hsa-miR-21, which has been shown to be up-regulated in active MS lesions [80] and to be higher expressed in PBMC of RRMS patients versus controls [81]. MicroRNAs in MS and therapy are worthy of being explored in more detail $[82,83]$. So far, there is only one study that has specifically investigated whether GA therapy affects the levels of mature microRNAs, but this study was limited to five selected microRNAs [84].

Other studies demonstrated that GA treatment leads to a change in the properties of monocytes from proinflammatory type I monocytes to anti-inflammatory type II monocytes [34]. However, although some cytokines were differentially expressed during GA therapy, the mRNA levels of TNF- $\alpha$, TGF- $\beta$, IL10, IL12, IL1- $\beta$ and IL1RA were not affected in our data set. Therefore, we could not observe a clear cytokine shift in monocytes in response to GA. One reason for that might be that in our study monocytes were isolated from peripheral blood samples of MS patients, whereas, in contrast, in the study by Weber et al. the monocytes were separated from the spleen of mice with EAE [34]. Moreover, our study was restricted to mRNA transcripts and we did not measure the amounts of the encoded proteins and their splice variants, whereas other groups analyzed the protein levels of monocytes in culture after in vitro stimulation [35,36,38]. Burger et al. studied the effects of GA on the transcription of two genes (IL1- $\beta$ and IL1RA) in monocytes $[39,40]$. However, their results might not be reflected in our data since they used monocytes from blood donors and stimulated these cells in vitro with GA. In our study, we could neither identify a stable signature of differentially expressed genes nor a solid evidence of an increase of type II monocytes within the first two months of therapy. This finding cannot be explained by just the relatively small number of recruited patients. Therefore, we conclude that the in vivo effects of GA on monocytes in the peripheral blood are rather modest and variable. It is likely that most of the effects occur at the injection sites or in the draining lymph nodes where (MBP-specific) T-cells as well as monocytes and professional APC are confronted with GA peptides. Additionally, since GA is a mixture of randomly 
synthesized peptides, the molecular effects might be somewhat different from injection to injection. All this makes it quite a challenge to study the drug's molecular mechanisms of action. Further studies are needed to better understand how GA modulates the immune system, also because new drugs similar to GA are currently tested for RRMS (for example, ATX-MS-1467, Apitope Technology Ltd., Bristol, UK).

\section{Conclusions}

There is a lack of transcriptome studies on the effects of GA in MS patients. Here, we presented the first genomewide and cell type-specific analysis of the mRNA dynamics during GA therapy. Using microarrays, we longitudinally measured the gene expression of monocytes for a small patient group at five different time points. We identified 463 genes as differentially expressed within the first two months of GA treatment, the majority being associated with immunological processes (for example, cytokines). However, the changes in gene expression were not sustained over time, and most genes were seen upregulated or down-regulated only once. Therefore, GA seems to have only little gene regulatory effects on monocytes. Our study nevertheless delivered some genes that are worth investigating in future studies regarding the molecular mechanisms of GA therapy in the peripheral blood of MS patients.

\section{Additional files}

Additional file 1: Figure S1. Analysis of the purity of the isolated monocytes. (A) We visualized the measured transcript levels of five selected genes, which are specifically expressed in different blood cell types, namely CD14 (monocytes), CD3D (T-cells), MS4A1 (for example, B-cells), KLRD1 (for example, NK cells) and HBD (erythrocytes). CD14 was expressed at very high levels (> 18,000) in all 37 samples of the microarray data set, whereas the other genes were expressed at very low levels $(<400)$. This demonstrates high purity of the monocytes isolated by MACS. (B) We used the Affymetrix microarray data by Novershtern et al. [59] to compare the mRNA levels of these genes in distinct human hematopoietic cell populations, for example, CD4+ and CD8+ T-cells, B-cells and monocytes. The preprocessed data were downloaded from the GEO database (accession number 'GSE24759'). The bar charts show the mean \pm standard error of the expression values of the respective probe sets (given in brackets) in 14 different cell types. A limited purity of the isolated monocytes would be noticeable in figure $A$, because CD3D, MS4A1, KLRD1 and HBD are highly expressed in other cells of the blood.

Additional file 2: Table S1. Filtered differentially expressed genes. This Excel file contains four gene lists, which provide the genes that were identified as differentially expressed after one day (t1), one week (t2), one month (t3) or two months (t4) of GA therapy when compared to baseline levels (t0). For each gene, identifiers for the databases GeneCards, Entrez and HGNC (gene symbols) are provided together with their official full names. Additionally, the mean gene expression levels (averaged over the patients) and the respective standard deviations (SD) are given for all compared time points as well as the computed t-test P-values and MAID-scores. The column 'Regulation' denotes, if the gene was found up-regulated or down-regulated in response to GA relative to baseline.

\section{Abbreviations}

APC: Antigen-presenting cell; APL: Altered peptide ligand; BP: Biological process; CC: Cellular component; CDF: Chip definition file; CNS: Central nervous system; DGN: German society of neurology; EAE: Experimental autoimmune encephalomyelitis; EDSS: Expanded disability status scale; EDTA: Ethylenediaminetetraacetic acid; FDR: False discovery rate; GA: Glatiramer acetate; GEO: Gene expression omnibus; GO: Gene ontology; HSP: Heat shock protein; IFN: Interferon; IL: Interleukin; MACS: Magnetic-activated cell sorting; MAID: MA plot-based signal intensity-dependent fold-change criterion; MBP: Myelin basic protein; MF: Molecular function; MHC: Major histocompatibility complex; MRI: Magnetic resonance imaging; MS: Multiple sclerosis; NK: Natural killer; PBMC: Peripheral blood mononuclear cells; RIN: RNA integrity number; RRMS: Relapsing-remitting multiple sclerosis; Sc: Subcutaneous; SD: Standard deviation; TCR: T-cell receptor; Th: T helper cell; Treg: T regulatory cell.

\section{Competing interests}

UKZ received research support as well as speaking fees from Teva, Biogen Idec, Bayer HealthCare, Novartis, Almirall, Merck Serono and Sanofi-Aventis. $\mathrm{MH}$ received speaking fees from Bayer HealthCare, Teva and Novartis. Meanwhile, RHG is a salaried employee of Teva, and he was a salaried employee of Novartis. MT, BKP, IS, DK and H-JT declare no potential conflict of interest.

\section{Authors' contributions}

UKZ and H-JT inspired and directed the work. IS was responsible for clinical documentation. The lab experiments were performed by RHG, DK, BKP and MT. MH and RHG carried out the analysis and interpretation of the data. MT and $\mathrm{MH}$ drafted the paper and prepared all tables and figures. H-JT and UKZ contributed to the writing of the manuscript. All authors read and approved the final manuscript.

\section{Acknowledgements}

The Affymetrix microarray experiments were partially funded by Teva Pharmaceutical Industries Ltd. We thank the United Europeans for the development of PHArmacogenomics in Multiple Sclerosis (UEPHA*MS) consortium, which is a Marie Curie Initial Training Network, for their funding. The funders had no role in study design, data collection, analysis and interpretation, decision to publish, or preparation of the manuscript. We thank Ildikó Tóth, Maximilian Fritsch, Robby Engelmann and Gabriele Gillwaldt for their help in performing the analyses. We thank Christa Tiffert for coordinating patient care and sample collection. We thank Karthik Sathiyanadan for providing literature necessary for this work.

Received: 21 August 2013 Accepted: 6 October 2013

Published: 17 October 2013

\section{References}

1. Barten LJ, Allington DR, Procacci KA, Rivey MP: New approaches in the management of multiple sclerosis. Drug Des Devel Ther 2010, 4:343-366.

2. Vosoughi R, Freedman MS: Therapy of MS. Clin Neurol Neurosurg 2010, 112(5):365-385.

3. Comi G, Martinelli V, Rodegher M, Moiola L, Bajenaru O, Carra A, Elovaara I, Fazekas F, Hartung HP, Hillert J, King J, Komoly S, Lubetzki C, Montalban X, Myhr KM, Ravnborg M, Rieckmann P, Wynn D, Young C, Filippi M, PreCISe study group: Effect of glatiramer acetate on conversion to clinically definite multiple sclerosis in patients with clinically isolated syndrome (PreCISe study): a randomized, double-blind, placebo-controlled trial. Lancet 2009, 374(9700):1503-1511.

4. Martinelli Boneschi F, Rovaris M, Johnson KP, Miller A, Wolinsky JS, Ladkani D, Shifroni G, Comi G, Filippi M: Effects of glatiramer acetate on relapse rate and accumulated disability in multiple sclerosis: meta-analysis of three double-blind, randomized, placebo-controlled clinical trials. Mult Scler 2003, 9(4):349-355.

5. Mikol DD, Barkhof F, Chang P, Coyle PK, Jeffery DR, Schwid SR, Stubinski B, Uitdehaag B, REGARD study group: Comparison of subcutaneous interferon beta-1a with glatiramer acetate in patients with relapsing multiple sclerosis (the REbif versus Glatiramer Acetate in Relapsing MS Disease [REGARD] study): a multicenter, randomized, parallel, open-label trial. Lancet Neurol 2008, 7(10):903-914. 
6. Simpson D, Noble S, Perry C: Spotlight on glatiramer acetate in relapsing-remitting multiple sclerosis. Bio Drugs 2003, 17(3):207-210.

7. Liblau R: Glatiramer acetate for the treatment of multiple sclerosis: evidence for a dual anti-inflammatory and neuroprotective role. J Neurol Sci 2009, 287(Suppl 1):S17-S23.

8. Mix E, Meyer-Rienecker $\mathrm{H}$, Hartung HP, Zettl UK: Animal models of multiple sclerosis - potentials and limitations. Prog Neurobiol 2010, 92(3):386-404.

9. Sela $M$, Teitelbaum D: Glatiramer acetate in the treatment of multiple sclerosis. Expert Opin Pharmacother 2001, 2(7):1149-1165.

10. Ziemssen T, Neuhaus $\mathrm{O}$, Hohlfeld R: Risk-benefit assessment of glatiramer acetate in multiple sclerosis. Drug Saf 2001, 24(13):979-990.

11. Farina C, Weber MS, Meinl E, Wekerle H, Hohlfeld R: Glatiramer acetate in multiple sclerosis: update on potential mechanisms of action. Lancet Neurol 2005, 4(9):567-575.

12. Karussis D, Teitelbaum D, Sicsic C, Brenner T, AC001 multi-center Israeli study group: Long-term treatment of multiple sclerosis with glatiramer acetate: natural history of the subtypes of anti-glatiramer acetate antibodies and their correlation with clinical efficacy. J Neuroimmunol 2010, 220(1-2):125-130.

13. Salama HH, Hong J, Zang YC, El-Mongui A, Zhang J: Blocking effects of serum reactive antibodies induced by glatiramer acetate treatment in multiple sclerosis. Brain 2003, 126(Pt 12):2638-2647.

14. Ure DR, Rodriguez M: Polyreactive antibodies to glatiramer acetate promote myelin repair in murine model of demyelinating disease. FASEB J 2002, 16(10):1260-1262.

15. Blanchette F, Neuhaus $O$ : Glatiramer acetate: evidence for a dual mechanism of action. J Neurol 2008, 255(Suppl 1):26-36.

16. Lalive PH, Neuhaus O, Benkhoucha M, Burger D, Hohlfeld R, Zamvil SS, Weber MS: Glatiramer acetate in the treatment of multiple sclerosis: emerging concepts regarding its mechanism of action. CNS Drugs 2011, 25(5):401-414

17. Neuhaus $\mathrm{O}$, Farina $\mathrm{C}$, Wekerle $\mathrm{H}$, Hohlfeld R: Mechanisms of action of glatiramer acetate in multiple sclerosis. Neurology 2001, 56(6):702-708.

18. Yong WW: Differential mechanisms of action of interferon-beta and glatiramer acetate in MS. Neurology 2002, 59(6):802-808

19. Aharoni R, Teitelbaum D, Arnon R, Sela M: Copolymer 1 acts against the immunodominant epitope 82-100 of myelin basic protein by $T$ cell receptor antagonism in addition to major histocompatibility complex blocking. Proc Natl Acad Sci USA 1999, 96(2):634-639.

20. Gran B, Tranquill LR, Chen M, Bielekova B, Zhou W, Dhib-Jalbut S, Martin R: Mechanisms of immunomodulation by glatiramer acetate. Neurology 2000, 55(11):1704-1714.

21. Hafler DA: Degeneracy, as opposed to specificity, in immunotherapy. J Clin Invest 2002, 109(5):581-584.

22. Ragheb S, Abramczyk S, Lisak D, Lisak R: Long-term therapy with glatiramer acetate in multiple sclerosis: effect on T-cells. Mult Scler 2001 7(1):43-47.

23. Schmied M, Duda PW, Krieger JI, Trollmo C, Hafler DA: In vitro evidence that subcutaneous administration of glatiramer acetate induces hyporesponsive T cells in patients with multiple sclerosis. Clin Immunol 2003, 106(3):163-174

24. Aharoni R, Teitelbaum D, Sela M, Arnon R: Bystander suppression of experimental autoimmune encephalomyelitis by $T$ cell lines and clones of the Th2 type induced by copolymer 1. J Neuroimmunol 1998, 91(1-2):135-146.

25. Aharoni R, Teitelbaum D, Leitner O, Meshorer A, Sela M, Arnon R: Specific Th2 cells accumulate in the central nervous system of mice protected against experimental autoimmune encephalomyelitis by copolymer 1 . Proc Natl Acad Sci USA 2000, 97(21):11472-11477.

26. Dhib-Jalbut S, Chen M, Said A, Zhan M, Johnson KP, Martin R: Glatiramer acetate-reactive peripheral blood mononuclear cells respond to multiple myelin antigens with a Th2-biased phenotype. J Neuroimmunol 2003, 140(1-2):163-171.

27. Neuhaus O, Farina C, Yassouridis A, Wiendl H, Then Bergh F, Dose T, Wekerle H, Hohlfeld R: Multiple sclerosis: comparison of copolymer-1reactive $T$ cell lines from treated and untreated subjects reveals cytokine shift from T helper 1 to Thelper 2 cells. Proc Natl Acad Sci USA 2000, 97(13):7452-7457.

28. Geissmann F, Manz MG, Jung S, Sieweke MH, Merad M, Ley K: Development of monocytes, macrophages, and dendritic cells. Science 2010, 327(5966):656-661.
29. Izikson L, Klein RS, Luster AD, Weiner HL: Targeting monocyte recruitment in CNS autoimmune disease. Clin Immunol 2002, 103(2):125-131.

30. Fridkis-Hareli M, Teitelbaum D, Gurevich E, Pecht I, Brautbar C, Kwon OJ, Brenner T, Arnon R, Sela M: Direct binding of myelin basic protein and synthetic copolymer 1 to class II major histocompatibility complex molecules on living antigen-presenting cells -specificity and promiscuity. Proc Natl Acad Sci USA 1994, 91(11):4872-4876.

31. Schrempf W, Ziemssen T: Glatiramer acetate: mechanisms of action in multiple sclerosis. Autoimmun Rev 2007, 6(7):469-475.

32. Vieira PL, Heystek HC, Wormmeester J, Wierenga EA, Kapsenberg ML: Glatiramer acetate (copolymer-1, copaxone) promotes Th2 cell development and increased IL-10 production through modulation of dendritic cells. J Immunol 2003, 170(9):4483-4488.

33. Weber MS, Starck M, Wagenpfeil S, Meinl E, Hohlfeld R, Farina C: Multiple sclerosis: glatiramer acetate inhibits monocyte reactivity in vitro and in vivo. Brain 2004, 127(Pt 6):1370-1378

34. Weber MS, Prod'homme T, Youssef S, Dunn SE, Rundle CD, Lee L, Patarroyo JC, Stüve O, Sobel RA, Steinman L, Zamvil SS: Type II monocytes modulate T cell-mediated central nervous system autoimmune disease. Nat Med 2007, 13(8):935-943.

35. Ayers CL, Mendoza JP, Sinha S, Cunnusamy K, Greenberg BM, Frohman EM, Karandikar NJ: Modulation of immune function occurs within hours of therapy initiation for multiple sclerosis. Clin Immunol 2013, 147(2):105-119.

36. Kim HJ, Ifergan I, Antel JP, Seguin R, Duddy M, Lapierre Y, Jalili F, Bar-Or A: Type 2 monocyte and microglia differentiation mediated by glatiramer acetate therapy in patients with multiple sclerosis. J Immunol 2004, 172(11):7144-7153

37. Li Q, Milo R, Panitch H, Swoveland P, Bever CT Jr: Glatiramer acetate blocks the activation of THP-1 cells by interferon-gamma. Eur J Pharmacol 1998, 342(2-3):303-310

38. Toker A, Slaney CY, Bäckström BT, Harper JL: Glatiramer acetate treatment directly targets $C D 11 \mathrm{~b}(+) \operatorname{Ly} 6 \mathrm{G}(-)$ monocytes and enhances the suppression of autoreactive $T$ cells in experimental autoimmune encephalomyelitis. Scand J Immunol 2011, 74(3):235-243.

39. Burger D, Molnarfi N, Weber MS, Brandt KJ, Benkhoucha M, Gruaz L, Chofflon M, Zamvil SS, Lalive PH: Glatiramer acetate increases IL-1 receptor antagonist but decreases T cell-induced IL-1beta in human monocytes and multiple sclerosis. Proc Natl Acad Sci USA 2009, 106(11):4355-4359.

40. Carpintero R, Brandt KJ, Gruaz L, Molnarfi N, Lalive PH, Burger D: Glatiramer acetate triggers PI3K $\delta / A k t$ and MEK/ERK pathways to induce IL-1 receptor antagonist in human monocytes. Proc Natl Acad Sci USA 2010, 107(41):17692-17697.

41. Caragnano M, Tortorella P, Bergami A, Ruggieri M, Livrea P, Specchio LM Martino G, Trojano M, Furlan R, Avolio C: Monocytes P2X7 purinergic receptor is modulated by glatiramer acetate in multiple sclerosis. J Neuroimmunol 2012, 245(1-2):93-97.

42. Tennakoon DK, Mehta RS, Ortega SB, Bhoj V, Racke MK, Karandikar NJ: Therapeutic induction of regulatory, cytotoxic CD8+ T cells in multiple sclerosis. J Immunol 2006, 176(11):7119-7129.

43. Mansilla MJ, Montalban X, Espejo C: Heat shock protein 70: roles in multiple sclerosis. Mol Med 2012, 18:1018-1028.

44. Nishikawa M, Takemoto S, Takakura Y: Heat shock protein derivatives for delivery of antigens to antigen presenting cells. Int J Pharm 2008, 354(1-2):23-27.

45. Aharoni R, Eilam R, Domev H, Labunskay G, Sela M, Arnon R: The immunomodulator glatiramer acetate augments the expression of neurotrophic factors in brains of experimental autoimmune encephalomyelitis mice. Proc Natl Acad Sci USA 2005, 102(52):19045-19050

46. Aharoni R, Herschkovitz A, Eilam R, Blumberg-Hazan M, Sela M, Bruck W, Arnon R: Demyelination arrest and remyelination induced by glatiramer acetate treatment of experimental autoimmune encephalomyelitis. Proc Natl Acad Sci USA 2008, 105(32):11358-11363.

47. Chen M, Valenzuela RM, Dhib-Jalbut S: Glatiramer acetate-reactive T cells produce brain-derived neurotrophic factor. J Neurol Sci 2003, 215(1-2):37-44.

48. Ruggieri M, Avolio C, Livrea P, Trojano M: Glatiramer acetate in multiple sclerosis: a review. CNS Drug Rev 2007, 13(2):178-191.

49. Skihar V, Silva C, Chojnacki A, Döring A, Stallcup WB, Weiss S, Yong W: Promoting oligodendrogenesis and myelin repair using the multiple 
sclerosis medication glatiramer acetate. Proc Natl Acad Sci USA 2009, 106(42):17992-17997.

50. Ziemssen T, Kümpfel T, Klinkert WE, Neuhaus O, Hohlfeld R: Glatiramer acetate-specific T-helper 1- and 2-type cell lines produce BDNF: implications for multiple sclerosis therapy. Brain-derived neurotrophic factor. Brain 2002, 125(Pt 11):2381-2391.

51. Goertsches RH, Zettl UK, Hecker M: Sieving treatment biomarkers from blood gene-expression profiles: a pharmacogenomic update on two types of multiple sclerosis therapy. Pharmacogenomics 2011, 12(3):423-432.

52. Achiron A, Feldman A, Gurevich M: Molecular profiling of glatiramer acetate early treatment effects in multiple sclerosis. Dis Markers 2009, 27(2):63-73.

53. Polman CH, Reingold SC, Edan G, Filippi M, Hartung HP, Kappos L, Lublin FD, Metz LM, McFarland HF, O'Connor PW, Sandberg-Wollheim M, Thompson AJ, Weinshenker BG, Wolinsky JS: Diagnostic criteria for multiple sclerosis: 2005 revisions to the 'McDonald Criteria'. Ann Neurol 2005, 58(6):840-846.

54. Ferrari F, Bortoluzzi S, Coppe A, Sirota A, Safran M, Shmoish M, Ferrari S, Lancet D, Danieli GA, Bicciato S: Novel definition files for human GeneChips based on GeneAnnot. BMC Bioinforma 2007, 8:446.

55. Hecker M, Goertsches RH, Engelmann R, Thiesen HJ, Guthke R: Integrative modeling of transcriptional regulation in response to antirheumatic therapy. BMC Bioinforma 2009, 10:262

56. Falcon S, Gentleman R: Using GOstats to test gene lists for GO term association. Bioinformatics 2007, 23(2):257-258.

57. Nikitin A, Egorov S, Daraselia N, Mazo I: Pathway studio-the analysis and navigation of molecular networks. Bioinformatics 2003, 19(16):2155-2157.

58. Cline MS, Smoot M, Cerami E, Kuchinsky A, Landys N, Workman C, Christmas R, Avila-Campilo I, Creech M, Gross B, Hanspers K, Isserlin R, Kelley R, Killcoyne S, Lotia S, Maere S, Morris J, Ono K, Pavlovic V, Pico AR, Vailaya A, Wang PL, Adler A, Conklin BR, Hood L, Kuiper M, Sander C, Schmulevich I, Schwikowski B, Warner GJ, et al: Integration of biological networks and gene expression data using Cytoscape. Nat Protoc 2007, 2(10):2366-2382.

59. Novershtern N, Subramanian A, Lawton LN, Mak RH, Haining WN, McConkey ME, Habib N, Yosef N, Chang CY, Shay T, Frampton GM, Drake AC, Leskov I, Nilsson B, Preffer F, Dombkowski D, Evans JW, Liefeld T, Smutko JS, Chen J, Friedman N, Young RA, Golub TR, Regev A, Ebert BL: Densely interconnected transcriptional circuits control cell states in human hematopoiesis. Cell 2011, 144(2):296-309.

60. Ottoboni L, Keenan BT, Tamayo P, Kuchroo M, Mesirov JP, Buckle GJ, Khoury SJ, Hafler DA, Weiner HL, De-Jager PL: An RNA profile identifies two subsets of multiple sclerosis patients differing in disease activity. Sci Trans/ Med 2012, 4(153):153ra131.

61. Benjamini $Y$, Hochberg $Y$ : Controlling the false discovery rate: a practical and powerful approach to multiple testing. J Royal Stat Soc 1995, 57:289-300.

62. Sanna A, Fois ML, Arru G, Huang YM, Link H, Pugliatti M, Rosati G, Sotgiu S: Glatiramer acetate reduces lymphocyte proliferation and enhances IL-5 and IL-13 production through modulation of monocyte-derived dendritic cells in multiple sclerosis. Clin Exp Immunol 2006, 143(2):357-362.

63. Sellebjerg F, Hesse D, Limborg S, Lund H, Søndergaard HB, Krakauer M, Sørensen PS: Dendritic cell, monocyte and T cell activation and response to glatiramer acetate in multiple sclerosis. Mult Scler 2013, 19(2):179-187.

64. Müller M, Carter S, Hofer MJ, Campbell IL: Review: The chemokine receptor CXCR3 and its ligands CXCL9, CXCL10 and CXCL11 in neuroimmunity - a tale of conflict and conundrum. Neuropathol Appl Neurobiol 2010, 36(5):368-387

65. Szczuciński A, Losy J: Chemokines and chemokine receptors in multiple sclerosis. Potential targets for new therapies. Acta Neurol Scand 2007, 115(3):137-146.

66. Hong J, Zang YC, Hutton G, Rivera VM, Zhang JZ: Gene expression profiling of relevant biomarkers for treatment evaluation in multiple sclerosis. J Neuroimmunol 2004, 152(1-2):126-139.

67. Goertsches RH, Hecker M, Zettl UK: Monitoring of multiple sclerosis immunotherapy: from single candidates to biomarker networks. J Neurol 2008, 255(Suppl 6):48-57.

68. Fitzgerald DC, Rostami A: Therapeutic potential of IL-27 in multiple sclerosis? Expert Opin Biol Ther 2009, 9(2):149-160.

69. Losy J, Niezgoda A: IL-18 in patients with multiple sclerosis Acta Neurol Scand 2001, 104(3):171-173.

70. Wang J, Fu YX: The role of LIGHT in T cell-mediated immunity. Immunol Res 2004, 30(2):201-214.

71. Malmeström C, Gillett A, Jernås M, Khademi M, Axelsson M, Kockum I, Mattsson N, Zetterberg H, Blennow K, Alfredsson L, Wadenvik H, Lycke J,
Olsson T, Olsson B: Serum levels of LIGHT in MS. Mult Scler 2013, 19(7):871-876

72. Sawcer S, Hellenthal G, Pirinen M, Spencer CC, Patsopoulos NA, Moutsianas L, Dilthey A, Su Z, Freeman C, Hunt SE, Edkins S, Gray E, Booth DR, Potter

SC, Goris A, Band G, Oturai AB, Strange A, Saarela J, Bellenguez C, Fontaine B, Gillman M, Hemmer B, Gwilliam R, Zipp F, Jayakumar A, Martin R, Leslie S, Hawkins S, Giannoulatou E, et al: Genetic risk and a primary role for cell-mediated immune mechanisms in multiple sclerosis. Nature 2011, 476(7359):214-219.

73. Ware CF: Network communications: lymphotoxins, LIGHT, and TNF. Annu Rev Immunol 2005, 23:787-819.

74. Ware CF, Sedý JR: TNF Superfamily Networks: bidirectional and interference pathways of the herpesvirus entry mediator (TNFSF14). Curr Opin Immunol 2011, 23(5):627-631.

75. Shi C, Simon DI: Integrin signals, transcription factors, and monocyte differentiation. Trends Cardiovasc Med 2006, 16(5):146-152.

76. Hermann P, Armant M, Brown E, Rubio M, Ishihara H, Ulrich D, Caspary RG, Lindberg FP, Armitage R, Maliszewski C, Delespesse G, Sarfati M: The vitronectin receptor and its associated CD47 molecule mediates proinflammatory cytokine synthesis in human monocytes by interaction with soluble CD23. J Cell Biol 1999, 144(4):767-775.

77. Ewalt KL, Schimmel P: Activation of angiogenic signaling pathways by two human tRNA synthetases. Biochemistry 2002, 41(45):13344-13349.

78. Hermiston ML, Xu Z, Weiss A: CD45: a critical regulator of signaling thresholds in immune cells. Annu Rev Immunol 2003, 21:107-137.

79. Kirchberger S, Majdic O, Blüml S, Schrauf C, Leitner J, Gerner C, Paster W, Gundacker N, Sibilia M, Stöckl J: The cytoplasmic tail of CD45 is released from activated phagocytes and can act as an inhibitory messenger for $T$ cells. Blood 2008, 112(4):1240-1248.

80. Junker A, Krumbholz M, Eisele S, Mohan H, Augstein F, Bittner R, Lassmann $H$, Wekerle $H$, Hohlfeld R, Meinl E: MicroRNA profiling of multiple sclerosis lesions identifies modulators of the regulatory protein CD47. Brain 2009, 132(Pt 12):3342-3352.

81. Fenoglio C, Cantoni C, De Riz M, Ridolfi E, Cortini F, Serpente M, Villa C, Comi C, Monaco F, Mellesi L, Valzelli S, Bresolin N, Galimberti D, Scarpini E: Expression and genetic analysis of miRNAs involved in CD4+ cell activation in patients with multiple sclerosis. Neurosci Lett 2011, 504(1):9-12.

82. Angerstein C, Hecker M, Paap BK, Koczan D, Thamilarasan M, Thiesen HJ, Zettl UK: Integration of MicroRNA databases to study MicroRNAs associated with multiple sclerosis. Mol Neurobio/ 2012, 45(3):520-535.

83. Hecker M, Thamilarasan M, Koczan D, Schröder I, Flechtner K, Freiesleben S, Füllen G, Thiesen HJ, Zettl UK: MicroRNA expression changes during interferon-beta treatment in the peripheral blood of multiple sclerosis patients. Int J Mol Sci 2013, 14(8):16087-16110.

84. Waschbisch A, Atiya M, Linker RA, Potapov S, Schwab S, Derfuss T: Glatiramer acetate treatment normalizes deregulated microRNA expression in relapsing remitting multiple sclerosis. PLoS One 2011, 6(9):e24604.

doi:10.1186/1742-2094-10-126

Cite this article as: Thamilarasan et al:: Glatiramer acetate treatment effects on gene expression in monocytes of multiple sclerosis patients. Journal of Neuroinflammation 2013 10:126

\section{Submit your next manuscript to BioMed Central and take full advantage of:}

- Convenient online submission

- Thorough peer review

- No space constraints or color figure charges

- Immediate publication on acceptance

- Inclusion in PubMed, CAS, Scopus and Google Scholar

- Research which is freely available for redistribution 